\title{
Quantum Anomalous Hall Effect by Coupling Heavy Atomic Layers with CrI ${ }_{3}$
}

\author{
Majeed Ur Rehman, ${ }^{1}$ Xinlong Dong, ${ }^{2,1}$ Tao Hou, ${ }^{1}$ Zeyu Li, ${ }^{1}$ Shifei Qi,${ }^{3,1}$ and Zhenhua Qiao ${ }^{1, *}$ \\ ${ }^{1}$ ICQD, Hefei National Laboratory for Physical Sciences at Microscale, \\ CAS Key Laboratory of Strongly-Coupled Quantum Matter Physics, and Department of Physics, \\ University of Science and Technology of China, Hefei, Anhui 230026, China. \\ ${ }^{2}$ College of Physics and Information Engineering, and Research Institute of Materials Science, \\ Shanxi Normal University, Linfen, Shanxi 041004, China. \\ ${ }^{3}$ Department of Physics, Hebei Normal University, Shijiazhuang, Hebei 050024, China.
}

(Dated: July 9, 2019)

\begin{abstract}
We explored the possibility of realizing quantum anomalous Hall effect by placing heavy-element atomic layer on top of monolayer $\mathrm{CrI}_{3}$ with a natural cleavage surface and broken time-reversal symmetry. We showed that $\mathrm{CrI}_{3} / \mathrm{X}(\mathrm{X}=\mathrm{Bi}, \mathrm{Sb}$, or $\mathrm{As})$ systems can open up a sizable bulk gap to harbour quantum anomalous Hall effect, e.g., $\mathrm{CrI}_{3} / \mathrm{Bi}$ is a natural magnetic insulator with a bulk gap of $30 \mathrm{meV}$, which can be further enlarged via strain engineering or adjusting spin orientations. We also found that the ferromagnetic properties (magnetic anisotropic energy and Curie temperature) of pristine $\mathrm{CrI}_{3}$ can be further improved due to the presence of heavy atomic layers, and the spin orientation can be utilized as a useful knob to tune the band structure and Fermi level of $\mathrm{CrI}_{3} / \mathrm{Bi}$ system. The topological nature, together with the enhanced ferromagnetism, can unlock new potential applications for $\mathrm{CrI}_{3}$-based materials in spintronics and electronics.
\end{abstract}

Introduction - The interplay of symmetry, spin-orbit coupling, and magnetic structure together helps realize various topological phases ranging from quantum Hall effect to topological superconductor. The quantum anomalous Hall effect or magnetic topological insulator corresponds to the quantum Hall effect without applying external magnetic field [1 3], and exhibits immense application potential in dissipationless quantum electronics [4 8]. Typically, an element possessing high spin-orbit coupling is usually magnetically inactive, and vice versa. Therefore, the magnetic topological insulator rarely exists in natural materials. Theoretically, many attempts have been implemented to make it realistic in a single system by doping/adsorption [8 18], chemical functionalization [19, 20], and heterostructure schemes [2125]. However, thus far, experimentally, the quantum anomalous Hall effect has been only observed in magnetic element-doped topological insulators at very low temperatures 26 -28]. It is noteworthy that a new temperature record of $3.5 \mathrm{~K}$ for observing the quantum anomalous Hall effect was reported in $\mathrm{MnBi}_{2} \mathrm{Te}_{4}$ system [29].

It has been proposed [30] that magnetic topological insulators may be realized by depositing heavy-element layers on top of the surface of non-van der Waals (nonvdW) magnetic insulators. However, such a strategy has several experimental complications, such as surface reconstruction due to existence of dangling bonds on the surface of substrates. Owing to the presence of strong chemical bonding between constituent layers, it is experimentally not easy to downsize the vertical dimension of non-vdW three-dimensional (3D) ferromagnetic/antiferromagnetic insulators while preserving the lateral surface geometry (or only changing it slightly). To push away all these practical barriers and make the ideal environment more realistic, here, we suggest a versatile exper- (a)

(b)

(c)

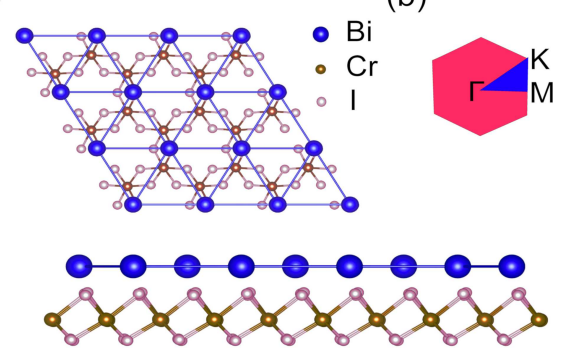

FIG. 1: (a) Crystal structure of Bi monolayer on monolayer $\mathrm{CrI}_{3}$ (labeled as $\mathrm{CrI}_{3} / \mathrm{Bi}$ ), and $\mathrm{Bi}$ atoms (blue spheres) form a triangular lattice over the substrate; (b) First Brillouin zone of $\mathrm{CrI}_{3} / \mathrm{Bi}$ along high symmetry lines; (c) Side view of $\mathrm{CrI}_{3} / \mathrm{Bi}$.

imental welcoming platform based on 2D vdW ferromagnetic insulators as a substrate for the deposition of heavy spin-orbit coupling components. Unlike a non-vdW substrate, 2D vdW ferromagnetic insulators, in principle, possess a natural cleavage plane with a perfect surface geometry free from dangling bonds, have no surface reform complication, are free of spin alignments, and are more accessible to being synthesized experimentally with excellent stability. This may attract the experimental community to invest their efforts in probing magnetic topological insulators. In addition, we address the following key queries to advance new, and possibly superior, routes for magnetic topological insulators. (i) Can the addition of spin-orbit coupling drive trivial $2 \mathrm{D} \mathrm{vdW}$ ferromagnetic systems to become large-bandgap magnetic topological insulators in a fundamental and controlled way? (ii) Can they improve the ferromagnetic properties of $2 \mathrm{D} v \mathrm{vdW}$ ferromagnetic insulators, such as the Curie temperature in these systems? 
In this Letter, we theoretically reported that several candidate systems $\mathrm{CrI}_{3} / \mathrm{X}(\mathrm{X}=\mathrm{Bi}, \mathrm{Sb}$, or $\mathrm{As})$ present non-trivial magnetic topological behaviors with a sizeable bandgap, by employing the state-of-art first-principles calculation methods. Besides a robust magnetic topology with non-zero Chern numbers, notable enhancements of the ferromagnetic properties of pristine $\mathrm{CrI}_{3}$ include the local magnetic moments, Heisenberg exchange interactions, and magnetic anisotropic energy and Curie temperature after coupling with heavy-atomic layers. Furthermore, we showed that either the ferromagnetic orientation or the strain effect can be utilized to tune the topologically nontrivial band gaps.

Ferromagnetic Properties of $\mathrm{CrI}_{3} / \mathrm{X}$ - We launched our search for stable configurations comprised of a heavyatoms monolayer, such as $\mathrm{Bi}, \mathrm{Sb}$, and $\mathrm{As}$ on the singlelayer ferromagnetic insulator, $\mathrm{CrI}_{3}$, to realize the quantum anomalous Hall effect in these systems. Upon a careful examination, we found that deposited heavy elements on $\mathrm{CrI}_{3}$ finally formed a monolayer with a triangular lattice structure, as displayed in Fig. 1(a). The evaluated interlayer binding energies for $\mathrm{CrI}_{3} / \mathrm{Bi}, \mathrm{CrI}_{3} / \mathrm{Sb}$, and $\mathrm{CrI}_{3} /$ As were $-0.65,-0.62$, and $-0.58 \mathrm{eV}$ per unitcell, respectively. This moderate interface interaction ensures that these atomic layers do not significantly affect the atomic structure of the pristine $\mathrm{CrI}_{3}$. To our surprise, we found a significant improvement in the magnetic moment of $\mathrm{Cr}$ in $\mathrm{CrI}_{3} / \mathrm{X}$ systems, as listed in Table 【 This is understandable, because the apparent interface interaction can change the charge distribution in $\mathrm{CrI}_{3}$ when heavy atomic layers are deposited on $\mathrm{CrI}_{3}$. As a result, the magnetic moments can be modified. This is indeed the case. Table \ displays that the charges of $\mathrm{Cr}$ in $\mathrm{CrI}_{3}$ and $\mathrm{CrI}_{3} / \mathrm{Bi}$ are 2.17 and $1.94 \mathrm{e}$, respectively, which indicate an additional 0.23 e transferring from $\mathrm{Bi}$ to $\mathrm{Cr}$ after the deposition of $\mathrm{Bi}$ monolayer. Same situation occurs for $\mathrm{CrI}_{3} / \mathrm{Sb}$ and $\mathrm{CrI}_{3} / \mathrm{As}$ systems. However, the magnitude of charge transfer decreases from $\mathrm{Bi}$ to As atomic layers. This is reasonable because the attraction ability of the nucleus on the valence electrons becomes weaker, and then, the probability of charge transfer is increased when we move from $\mathrm{As}$ to $\mathrm{Bi}$ in the periodic table. Thus, the magnetic moment of $\mathrm{Cr}$ atoms in a $\mathrm{CrI}_{3}$ single layer can be obviously enlarged by coupling with heavy atomic layers.

By depositing heavy atomic layers, the local magnetic moments of $\mathrm{Cr}$ in $\mathrm{CrI}_{3}$ are considerably increased. However, what about the magnetic coupling between these enhanced magnetic moments? We found that the resulting ferromagnetic coupling in $\mathrm{CrI}_{3}$ can also be enhanced. From our calculations, the ferromagnetic stabilities were $\triangle E_{\mathrm{FM}-\mathrm{AFM}}=-130,-96$ and $-86 \mathrm{meV}$ for $\mathrm{CrI}_{3} / \mathrm{Bi}, \mathrm{CrI}_{3} / \mathrm{Sb}$ and $\mathrm{CrI}_{3} / \mathrm{As}$ systems, respectively, where $\triangle E_{\mathrm{FM}-\mathrm{AFM}}$ represents the energy difference between ferromagnetic and anti-ferromagnetic arrangements. This indicates that the ferromagnetic nature of monolayer $\mathrm{CrI}_{3}$ can
TABLE I: Bader charge analysis for $\mathrm{CrI}_{3} / \mathrm{X}$ systems. $\triangle \mathrm{Q}$ represents the charge difference on $\mathrm{Cr}$ and iodine after the deposition of heavy atomic layers on $\mathrm{CrI}_{3}$ monolayer. Magnetic moments of each Cr atom, Heisenberg exchange constants, and Curie temperatures are symbolized by $M, J$, and $T_{\mathrm{C}}$ for $\mathrm{CrI}_{3} / \mathrm{X}$ systems, respectively.

\begin{tabular}{c|c|c|c|c|c}
\hline \multirow{2}{*}{ System } & \multicolumn{2}{|c|}{$\triangle \mathrm{Q}$} & \multicolumn{3}{c}{ Magnetic Properties } \\
\cline { 2 - 6 } & $\mathrm{Cr}$ & $\mathrm{I}$ & $M_{\mathrm{Cr}}\left(\mu_{B}\right)$ & $J(\mathrm{meV})$ & $T_{\mathrm{C}}(\mathrm{K})$ \\
\hline $\mathrm{CrI}_{3}$ & 0 & 0 & 3.0 & 2.59 & 44 \\
\hline $\mathrm{CrI}_{3} / \mathrm{Bi}$ & 0.23 & 0.27 & 3.72 & 9.62 & 165 \\
\hline $\mathrm{CrI}_{3} / \mathrm{Sb}$ & 0.21 & 0.24 & 3.70 & 7.10 & 122 \\
\hline $\mathrm{CrI}_{3} / \mathrm{As}$ & 0.11 & 0.14 & 3.62 & 6.37 & 110 \\
\hline \hline
\end{tabular}

be further stabilized by interacting with $\mathrm{X}(=\mathrm{Bi}, \mathrm{Sb}, \mathrm{As})$ atomic layers. In principle, the improvement of ferromagnetic stability boosts the Heisenberg exchange constant $J$. Our estimated Heisenberg exchange constants are respectively $9.62,7.10$, and $6.37 \mathrm{meV}$ for $\mathrm{Cr} I_{3} / \mathrm{Bi}$, $\mathrm{CrI}_{3} / \mathrm{Sb}$, and $\mathrm{CrI}_{3} / \mathrm{As}$. These are much higher than that of monolayer $\mathrm{CrI}_{3}(\sim 2.59 \mathrm{meV})$ [31]. Based on the above data and the mean field theory, the estimated Curie temperatures are 165,122 , and $110 \mathrm{~K}$ for $\mathrm{CrI}_{3} / \mathrm{Bi}, \mathrm{CrI}_{3} / \mathrm{Sb}$, and $\mathrm{CrI}_{3} / \mathrm{As}$, respectively 32]. These are greatly enhanced from $\sim 44 \mathrm{~K}$ of monolayer $\mathrm{CrI}_{3}$. In particular, a high Curie temperature is tall-demandable for quantum anomalous Hall system to operate at moderate temperatures in future device applications.

After determining that $\mathrm{CrI}_{3} / \mathrm{X}$ systems favor the ferromagnetic ground state, we then proceeded to investigate the magnetic anisotropy. Our results showed that $z$ axis is an easy magnetization direction for the $\mathrm{CrI}_{3} / \mathrm{Bi}$ system, which is approximately $5 \mathrm{meV}$ (per unit-cell with two $\mathrm{Cr}$ atoms) lower than that of $x$ axis. This value is much higher than that of monolayer $\mathrm{CrI}_{3}$ $(\sim 1.5 \mathrm{meV})$. This obvious improvement in magnetic anisotropic energy arises from the strong spin-orbit coupling in $\mathrm{CrI}_{3} / \mathrm{Bi}$, which has been reported in various systems [33, 34].

Quantum Anomalous Hall Effect-. The enhancement in ferromagnetic properties of pristine $\mathrm{CrI}_{3}$, including the local magnetic moments, Heisenberg exchange interactions, magnetic anisotropic energy and Curie temperature, will be beneficial to realizing the quantum anomalous Hall effect in $\mathrm{CrI}_{3}$ after coupling with heavyatomic layers. Figure 2(a) displays the spin-polarized band structure of $\mathrm{CrI}_{3} / \mathrm{Bi}$. The spin-up and -down bands cross around $\Gamma$ to form $\mathrm{D}_{1 / 2}$ points, which are closely located near the Fermi level. Considering the presence of $\mathrm{Bi}$ states at the Fermi level 32], together with the conceptual framework of magnetic topological 


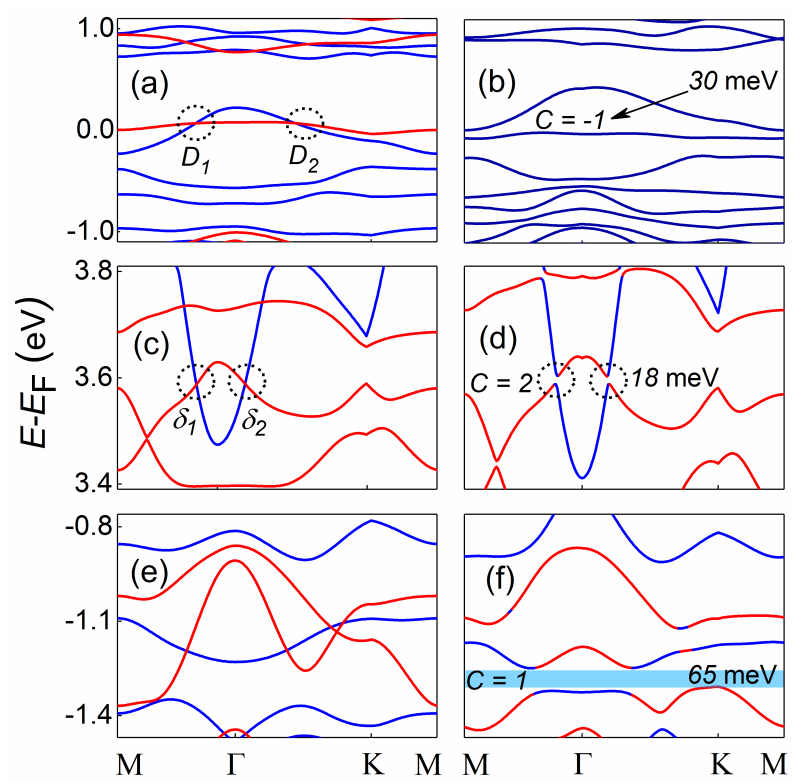

FIG. 2: (a-c-e): Spin-polarized band structures of $\mathrm{CrI}_{3} / \mathrm{Bi}$ (a), $\mathrm{CrI}_{3} / \mathrm{As}$ (c) and $\mathrm{CrI}_{3} / \mathrm{Sb}(\mathrm{e})$, respectively. (b-d-f): Band structures with spin-orbit coupling of $\mathrm{CrI}_{3} / \mathrm{Bi}$ (b), $\mathrm{CrI}_{3} / \mathrm{As}$ (d) and $\mathrm{CrI}_{3} / \mathrm{Sb}$ (f), respectively. Blue and red colors denote spin-up and -down bands.

insulators, one may expect the formation of topological non-trivial insulator around these avoided band-crossing points. When spin-orbit coupling is further considered in $\mathrm{CrI}_{3} / \mathrm{Bi}$, the degenerate states at the avoided bandcrossing points repel each other to open up an energy gap (see Fig. 2(b)). Our achieved direct and indirect (global) bandgaps in the $\mathrm{CrI}_{3} / \mathrm{Bi}$ are respectively 30 and $75 \mathrm{meV}$. We also confirmed the non-trivial behavior inside the band gap by calculating the Chern number using the approach implemented in Wannier90 and WannierTools packages [35, 36]. Our calculations demonstrate that $\mathrm{CrI}_{3} / \mathrm{Bi}$ indeed harbours a quantum anomalous Hall effect associated with a nonzero Chern number of $\mathcal{C}=-1$ [37].

Figure 2(c) displays the spin-polarized band structure for $\mathrm{CrI}_{3} /$ As. We found that the spin-up band crosses the spin-down bands twice within the energy window from 3.4 to $3.7 \mathrm{eV}$ around $\Gamma$ point. These band-crossing points are labeled as $\delta_{1 / 2}$. Interestingly, the band structures around these crossing points resemble the ideal cone (Dirac point) in graphene. Due to the relatively strong spin-orbit coupling of As, one may expect a non-trivial bandgap to open around these Dirac points when spinorbit coupling is invoked. Figure 2(d) presents the band structure in the presence of spin-orbit coupling, where bandgaps open up around the crossing points of $\delta_{1 / 2}$, which clearly indicate the existence of non-trivial band topologies in the conduction bands of $\mathrm{CrI}_{3} / \mathrm{As}$. By using the above mentioned approach, we obtained the Chern number of $\mathcal{C}=2$ for the corresponding opened band gaps. Additionally, the two valence bands touch each other around $\mathrm{K}$ point [32]. When the spin-orbit coupling is switched on, bandgaps are opened at these touching points, which signifies the topological nature of the $\mathrm{CrI}_{3} /$ As in valence bands. In principle, the non-trivial local bandgap in the conduction bands of $\mathrm{CrI}_{3} / \mathrm{As}$ can be converted into an insulator by charge doping technique, which is equivalent to the electrostatic gating in experiment. To tune the Fermi level into the conduction band, we applied a homogenous background charge to electrostatically control the electron doping using the self-consistent simulation approach. We noticed that the Fermi level was correctly located into the spin-orbit coupling induced bandgap without affecting the band structure pattern when the homogeneous charge of $\triangle Q=1 \mathrm{e}$ was functionalized 32. Notably, the non-trivial bandgap is also widened from 18 to $37 \mathrm{meV}$ upon the functionalization of electrostatic doping [32]. Thus, $\mathrm{CrI}_{3} / \mathrm{As}$ can also become a quantum anomalous Hall insulator upon moderate electrostatic gating.

Figure 2(e) displays the spin-polarized band structure of $\mathrm{CrI}_{3} / \mathrm{Sb}$. The spin-up and -down bands cross each other multiple times in the energy range between -1.30 and $-1.15 \mathrm{eV}$. A non-trivial bandgap opens at these crossing points upon the application of spin-orbit coupling [see Fig. 2(f)]. The global bandgap is approximately $65 \mathrm{meV}$, and exhibits a non-trivial topological nature with a Chern number of $\mathcal{C}=1$. Similar electrostatic gating approach can be used to tune the Fermi level into the bandgap.

Spin Orientation Effect-. The orientation of spin moments along different crystallographic directions provides one way to control the band structure and its associated topologically non-trivial properties. In a broader context, a strong spin-orbit coupling, together with a less symmetric crystallographic structure and a suitable magnetic behavior, is the main parameter to explore the spin orientation effect. Fortunately, $2 \mathrm{D} \mathrm{CrI}_{3} / \mathrm{X}$ systems fulfill all these criteria, and hence, are promising candidate platforms for demonstrating the spin orientation effects. In Fig. 3, our calculations show that tuning the spin moment from $c$ to $a$ axis in $\mathrm{CrI}_{3} / \mathrm{Bi}$ system remarkably modifies the band structures, including substantially changing the Fermi surface and bandgaps. In particular, one can observe that when all spin moments were adjusted in a ferromagnetic pattern from $0^{\circ}$ to ${ }^{6} 80$, the bandgap first gradually increases and then quickly decreases to be closed. Beyond $\theta=68 \circ$, the $\mathrm{CrI}_{3} / \mathrm{Bi}$ system undergos a topological phase transition from quantum anomalous Hall effect to a magnetic metallic state.

Strain Effect-. Strain is one of the significant approaches for engineering electronic and topological properties. Fortunately, the softness level of monolayer $\mathrm{CrI}_{3}$ is higher than that of graphene and $\mathrm{MoS}_{2}$, indicating that monolayer $\mathrm{CrI}_{3}$ can be easily stretched or compressed experimentally 38]. From this perspective, we examined the $\mathrm{CrI}_{3} / \mathrm{Bi}$ system under the application of sev- 


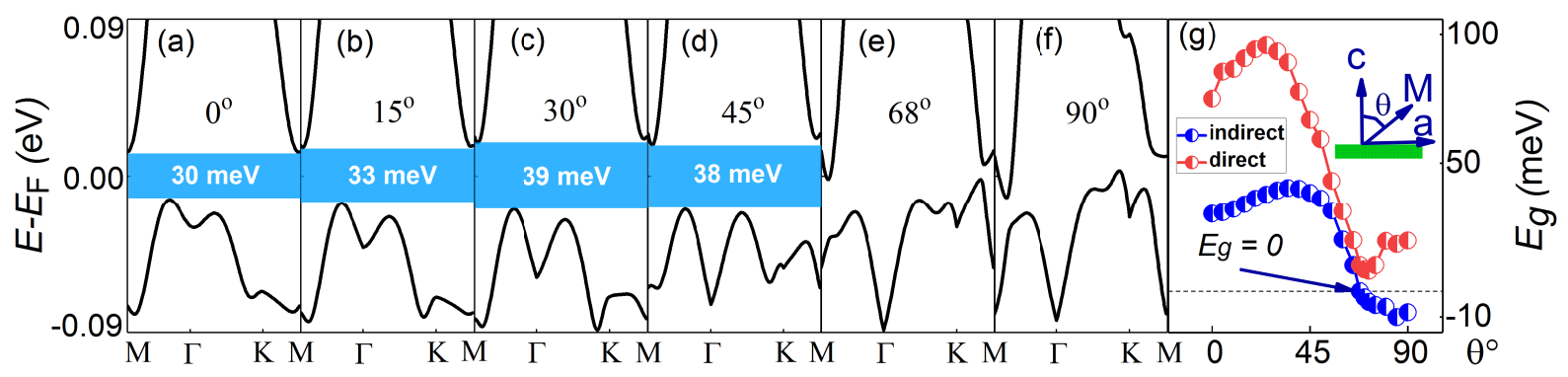

FIG. 3: Effect of spin orientation on the band structure of the $\mathrm{CrI}_{3} / \mathrm{Bi}$ system with $\mathrm{SOC}$ effects included. (a)-(f) present the SOC-included band structure when all spins are aligned at angles of $0^{\circ}, 15^{\circ}, 30^{\circ}, 45^{\circ}, 68^{\circ}$, and $90^{\circ}$, respectively. $(\mathrm{g})$ The variation in the direct and indirect bandgaps in $\mathrm{CrI}_{3} / \mathrm{Bi}$ as a function of $\theta$, where angle $\theta$ is measured in degrees from the vertical axis. The blue and red curves correspond to the indirect and direct bandgaps, respectively.

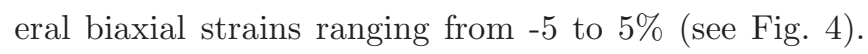
We observed that both direct and indirect bandgaps are much sensitive to the strain. In the upper panels, we considered the out-of-plane magnetization along the vertical direction with $\theta=0^{\circ}$. It is observed that the global bandgap considerably increases when the lattice is allowed to compress within the horizontal plane. Interestingly, the nature of the global bandgap changes from indirect to direct at an approximately $3.15 \%$ compressive strain (see Fig. 4). While for the in-plane magnetization with $\theta=90^{\circ}$ [see lower panels], one can find the the global bandgap remains closed in the typical range of tensile strains. Similarly, the global bandgap remains zero against the compressive strain. However, surprisingly, the zero nature of global bandgap converts into a nonzero one when the compressive strain is increased beyond $4 \%$, as shown in Fig. 4(h). This bandgap progressively enlarges upon a further increase in compressive strain. Therefore, in $\mathrm{CrI}_{3} / \mathrm{Bi}$, the quantum anomalous Hall effect with a Chern number of $\mathcal{C}=1$ can also be realized even in the presence of in-plane magnetization at a compressive strain slightly larger than $4 \%$.

Substrate Prototype- Finally, we considered $\mathrm{CrI}_{3} / \mathrm{Bi}$ as a representative example to search for an experimentally suitable non-magnetic substrate that guarantees the survival of Dirac points around the Fermi level. We found that $\mathrm{MoTe}_{2}$ (001) can serve as a good non-magnetic substrate 32]. Our projected principles for discovering quantum anomalous Hall materials can be straightforwardly extended to other vdW 2D ferromagnetic systems. Because of the epitaxial techniques, it becomes much more convenient to artificially enlarge the family of $2 \mathrm{D} \mathrm{vdW}$ ferromagnetic materials. Compared to traditional spintronic devices that are comprised of magnetic materials with some finite thickness, vdW ferromagnetic materials can simplify the device structure and shrink the device dimensions.

Summary-. Based on density functional theory, we confirmed that $\mathrm{CrI}_{3} / \mathrm{Bi}$ system is a quantum anomalous Hall insulator with a direct bandgap of approximately

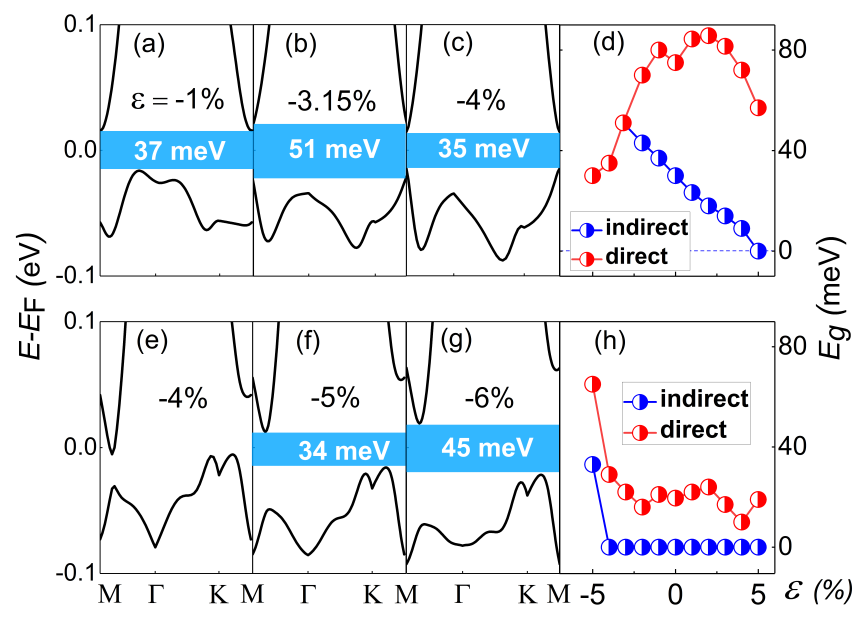

FIG. 4: (a)-(d) Band structures with out-of-plane magnetization in the presence of compressive strains, i.e., -1\% (a), $3.15 \%$ (b), and $-4 \%$ (c); (d) Bandgaps (direct and indirect) as function of compressive strain. (e)-(h) Band structures with in-plane magnetization in the presence of compressive strains, i.e., $-4 \%$ (e), $-5 \%$ (f), and $-6 \%(\mathrm{~g})$; (h) Bandgaps (direct and indirect) as function of compressive strain. $\varepsilon$ symbolizes the strain in \%.

$30 \mathrm{meV}$ at the Fermi level. We observed that $\mathrm{CrI}_{3} / \mathrm{As}$ and $\mathrm{CrI}_{3} / \mathrm{Sb}$ can open up topologically nontrivial band gaps in their conduction and valence bands, which can be properly tuned close the Fermi level via moderate electrostatic gating. Apart from the stable topology, we also found a major development in the ferromagnetic properties of $\mathrm{CrI}_{3}$, including local magnetic moments, Heisenberg exchange interactions, magnetic anisotropic energy, and Curie temperatures by coupling with atomic layers of $\mathrm{Bi} / \mathrm{As} / \mathrm{Sb}$. Furthermore, we showed that the band gaps of our proposed systems can be efficiently manipulated via tuning spin orientation or applying strain. Our study of $\mathrm{CrI}_{3} / \mathrm{X}$ system can open new routes to explore quantum anomalous Hall effect based on 2D ferromagnetic insulators together with the potential application of $\mathrm{CrI}_{3}$-based materials in spintronics. 
Acknowledgements - . This work was financially supported by the National Key R \& D Program (2017YFB0405703), the NNSFC (11474265), and Anhui Initiative in Quantum Information Technologies. We also thank the supercomputing service of AMHPC and the Supercomputing Center of USTC for providing the highperformance computing resources.

* Correspondence author: qiao@ustc.edu.cn

[1] H. Weng, R. Yu, X. Hu, X. Dai, and Z. Fang, Adv. Phys. 64, 227 (2015)

[2] Y. Ren, Z. Qiao, and Q. Niu, Rep. Prog. Phys. 79, 066501 (2016).

[3] K. He, Y. Wang, and Q.-K. Xue, Annu. Rev. Condens. Matter Phys. 9, 329 (2018).

[4] Z. Liu, G. Zhao, B. Liu, Z. Wang, J. Yang, and F. Liu, Phys. Rev. Lett. 121, 246401 (2018).

[5] X. Ni, W. Jiang, H. Huang, K.-H. Jin, and F. Liu, Nanoscale 10, 11901 (2018).

[6] Z. Zanolli, C. Niu, G. Bihlmayer, Y. Mokrousov, P. Mavropoulos, M. Verstraete, and S. Blugel, Phys. Rev. B 98, 155404 (2018).

[7] Y. Ren, T.-S. Zeng, W. Zhu, and D. Sheng, Phys. Rev. B 98, 205146 (2018).

[8] X. Deng, H. Yang, S. Qi, X. Xu, and Z. Qiao, Front. Phys. 13, 137308 (2018).

[9] W.-K. Tse, Z. Qiao, Y. Yao, A. MacDonald, and Q. Niu, Phys. Rev. B 83, 155447 (2011).

[10] Z. Qiao, S. A. Yang, W. Feng, W.-K. Tse, J. Ding, Y. Yao, J. Wang, and Q. Niu, Phys. Rev. B 82, 161414 (2010).

[11] J. Ding, Z. Qiao, W. Feng, Y. Yao, and Q. Niu, Phys. Rev. B 84, 195444 (2011).

[12] H. Zhang, C. Lazo, S. Blugel, S. Heinze, and Y. Mokrousov, Phys. Rev. Lett. 108, 056802 (2012).

[13] Z. Qiao, H. Jiang, X. Li, Y. Yao, and Q. Niu, Phys. Rev. B 85, 115439 (2012).

[14] H. Jiang, Z. Qiao, H. Liu, and Q. Niu, Phys. Rev. B 85, 045445 (2012).

[15] X. Kou, M. Lang, Y. Fan, Y. Jiang, T. Nie, J. Zhang, W. Jiang, Y. Wang, Y. Yao, L. He, et al., Acs Nano 7, 9205 (2013).

[16] H. Pan, Z. Li, C.-C. Liu, G. Zhu, Z. Qiao, and Y. Yao, Phys. Rev. Lett. 112, 106802 (2014).
[17] S. Qi, Z. Qiao, X. Deng, E. D. Cubuk, H. Chen, W. Zhu, E. Kaxiras, S. Zhang, X. Xu, and Z. Zhang, Phys. Rev. Lett. 117, 056804 (2016).

[18] X. Deng, S. Qi, Y. Han, K. Zhang, X. Xu, and Z. Qiao, Phys. Rev. B 95, 121410 (2017).

[19] K.-H. Jin and S.-H. Jhi, Sci. Rep. 5, 8426 (2015).

[20] C.-H. Hsu, Y. Fang, S. Wu, Z.-Q. Huang, C. P. Crisostomo, Y.-M. Gu, Z.-Z. Zhu, H. Lin, A. Bansil, F.-C. Chuang, et al., Phys. Rev. B 96, 165426 (2017).

[21] W. Luo and X.-L. Qi, Phys. Rev. B 87, 085431 (2013).

[22] H.-X. Yang, A. Hallal, D. Terrade, X. Waintal, S. Roche, and M. Chshiev, Phys. Rev. Lett. 110, 046603 (2013).

[23] Z. Qiao, W. Ren, H. Chen, L. Bellaiche, Z. Zhang, A. MacDonald, and Q. Niu, Phys. Rev. Lett. 112, 116404 (2014).

[24] G. Xu, J. Wang, C. Felser, X.-L. Qi, and S.-C. Zhang, Nano Lett. 15, 2019 (2015).

[25] J. Zhang, B. Zhao, T. Zhou, Y. Xue, C. Ma, and Z. Yang, Phys. Rev. B 97, 085401 (2018).

[26] C.-Z. Chang, J. Zhang, X. Feng, J. Shen, Z. Zhang, M. Guo, K. Li, Y. Ou, P. Wei, L.-L. Wang, et al., Science 340, 167 (2013).

[27] C.-X. Liu, S.-C. Zhang, and X.-L. Qi, Annu Rev. Condens. Matter Phys. 7, 301 (2016).

[28] A. Bestwick, E. Fox, X. Kou, L. Pan, K. L. Wang, and D. Goldhaber-Gordon, Phys. Rev. Lett. 114, 187201 (2015).

[29] Y. Deng, Y. Yu, M. Z. Shi, J. Wang, X. H. Chen, and Y. Zhang, arXiv:1904.11468 (2019).

[30] K. F. Garrity and D. Vanderbilt, Phys. Rev. Lett. 110, 116802 (2013).

[31] L. Webster and J.-A. Yan, Phys. Rev. B 98, 144411 (2018).

[32] See details in Supplemental Materials.

[33] O. Újsághy, A. Zawadowski, and B. L. Gyorffy, Phys. Rev. Lett. 76, 2378 (1996).

[34] D. Yi, J. Liu, S.-L. Hsu, L. Zhang, Y. Choi, J.-W. Kim, Z. Chen, J. D. Clarkson, C. R. Serrao, E. Arenholz, et al., PNAS 113, 6397 (2016).

[35] A. A. Mostofi, J. R. Yates, Y.-S. Lee, I. Souza, D. Vanderbilt, and N. Marzari, Comput. Phys. Commun. 178, 685 (2008).

[36] Q. Wu, S. Zhang, H.-F. Song, M. Troyer, and A. A. Soluyanov, Comput. Phys. Commun. 224, 405 (2018).

[37] D. J. Thouless, M. Kohmoto, M. P. Nightingale, and M. den Nijs, Phys. Rev. Lett. 49, 405 (1982).

[38] F. Zheng, J. Zhao, Z. Liu, M. Li, M. Zhou, S. Zhang, and P. Zhang, Nanoscale 10, 14298 (2018). 